\title{
Cost-Efficient NGN Rollout
}

\author{
Sofie Verbrugge, Jan Van Ooteghem, Koen Casier, \\ Marlies Van der Wee, and Mathieu Tahon \\ Ghent University, IBBT, \\ Dept. of Information Technology (INTEC) \\ \{sofie.verbrugge, jan.vanooteghem, koen. casier, \\ marlies.vanderwee, mathieu.tahon\} @intec.ugent.be
}

\begin{abstract}
We introduce in this section potential business models for the deployment of optic fiber networks. Because Fiber-to-the-Home is deployed at a slower speed than expected, we describe the evolution in several European countries, identify the reasons of the differences, propose a holistic approach to improve the business, and present a game-theoretic model to evaluate the impact of municipality investments on the market.
\end{abstract}

Keywords: NGN rollout, value network, holistic approach, game-theory.

\section{Introduction}

The rollout of new fiber access networks or the upgrade towards Fiber-to-the-Home (FTTH) is happening in most Western countries at a much slower rate than expected. The final step in migrating to an all fiber (last mile) access network is one bridge too far for most telecom operators. Nevertheless, FTTH is considered as the solution for access networks in the long run. In order to analyze the different technical and business aspects regarding future proof strategies for the rollout of FTTH, a strong focus must be put on the business and techno-economic evaluation.

This section will focus on three main issues within the techno-economic domain of networks. The first paragraph will describe which business models and value networks can be used to deploy FTTH networks, focusing on the different actors involved (both public and private) and the role each of them plays in the deployment and operations of the network. Several existing European networks will be analyzed and compared, to identify the best practices for the rollout of FTTH. Secondly, we will focus on the deployment itself, and identify a holistic approach that can improve the viability of the business case and lead to an earlier deployment and higher coverage. In the third and final section of this section, the influence of competition on the viability of the business case will be investigated, by applying a game-theoretic approach on a case in which a municipality rolls out FTTH in competition with another network operator upgrading its infrastructure. 


\section{Business Models and Value Networks for a Successful FTTH Deployment}

To identify best practices for the rollout of a new FTTH network, we start by setting up a framework to analyze existing networks in Europe, focusing on actors involved and the roles they take up. This framework, the so-called network matrix, will be explained first and later in this section used to identify different business models.

\subsection{Network Matrix}

The network matrix describes the different roles within the deployment and operations of an FTTH network (Figure 1, based on [9]). The different lifecycle phases of the network (deployment, provisioning of the early subscribers together with deployment, provisioning later on and operations) are found on the x-axis, while the $y$-axis shows the different parts of the network (backbone, access, building and home). Within each cell of the matrix, several boxes represent the different network layers, which can be easily interpreted as an extension of the classical OSI-model [5] towards fiber-based networks.

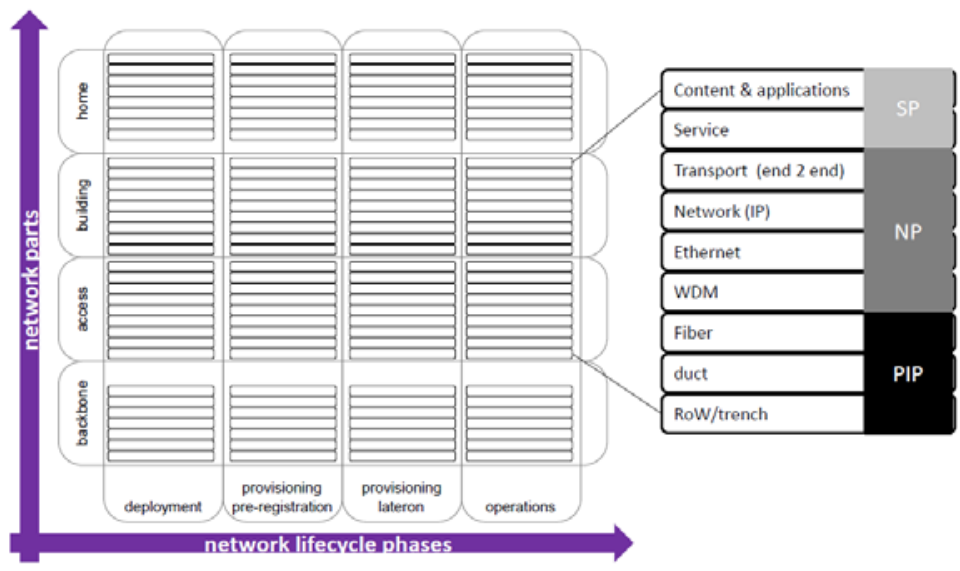

Fig. 1. The Network Matrix indicating all the roles

Focusing on the different network layers within each cell of the matrix allows identifying three important roles, which will be used in the next paragraph to identify the possible business models.

The Physical Infrastructure Provider (PIP) is responsible for obtaining Right of Way (RoW, the right to open up the streets) and performing the digging works itself, installing ducts and fibers or blowing the fibers afterwards. The PIP can also take care of other passive infrastructure, such as the housing of the Central Office (CO), installation of empty racks, provisioning of man- and hand-holes and so on. 
The Network Provider (NP) deploys and operates all the active equipment necessary to provide an end-to-end connectivity between the customers and the CO. They install specific equipment at the CO (Optical Line Terminal - OLT) and at the customer's premises (Optical Network Unit - ONU) and are responsible for the other network equipment (like switches, splitters...) in between.

When an end-to-end connectivity is present, the Service Provider (SP) can use the active network to offer services. His responsibility is to install the service-specific equipment (e.g., a set-top box for digital television) and to send the right content and applications to its subscribers. It is important to mention here that we consider only the local service providers that receive direct revenues from their customers through subscriptions and not the over the top players like Google etc. that get their main income out of advertising.

\subsection{Business Models}

One of the most important issues when analyzing an FTTH deployment is the identification of the business model: who is responsible for which role and does this mapping of actors to roles allow for competition. This paragraph gives a short explanation of the different business models encountered while analyzing the different cases, so they can easily be referred to in the next sections.

Based on theoretical studies and experience from experts in the field, we identified two possibilities for opening up a network to competition: on network level and service level [4]. From these opportunities for competition, several business models can be extracted, but we will only focus here on those that we encountered in analyzing the cases. The first business model is the Vertically Integrated one, in which one actor (or its subsidiaries) takes up all the roles. The Open Access model with competition on service level is characterized by one single PIP, one NP and several SP's. End-customers are then able to subscribe to services from different service providers, but the provisioning of end-to-end connectivity is a monopoly. The third and last model, Equal Open Access, allows for competition on both network and service level, since multiple NP's and multiple SP's are operating on top of one passive network. In this case, price competition can be combined with speed differentiation, because NP's are responsible for the offered bandwidth.

\subsection{Existing European Network Deployments}

In Europe, there are several networks already deployed and in an operational phase. This paragraph will compare different FTTH networks based on the business model they use, based on the analysis performed in [8]. The cases are subdivided in three types: urban, rural and large-scale deployments, because of the differences in type of rollout and presence of other access networks, like DSL or cable.

\section{Urban Regions}

Urban regions are characterized by the presence of other broadband networks, like the DSL network of the incumbent or an alternative network owned by a cable operator. 
This might be one of the reasons why we see here a clear preference towards the (Equal) Open Access Business Model, allowing room for competition resulting in a reduction of prices charged.

In urban regions, it is mostly the city (or a publicly owned utility company) that recognized the need for a fiber network and initiated the venture. In general, the main reason for deploying the network comes down to boosting the local economy and the ICT market, as well as increasing competition. Apart from the public entity (city or utility), housing corporations and private investors seem to have a rather big influence, both financially and in aggregation of demand. In Amsterdam for instance, the housing companies took up $1 / 3^{\text {rd }}$ of the initial investment $\left(1 / 3^{\text {rd }}\right.$ was taken up by the city of Amsterdam, $1 / 3^{\text {rd }}$ by private banks), meanwhile ensuring a certain take-up rate from the start, as all their homes got connected. Key motivations for the housing corporations to invest in FTTH networks were two-fold: being able to offer a fast and reliable FTTH connection to their residents, which in turn increases the value of their real-estate property.

\section{Large-Scale Deployments}

The second category of European cases under consideration is that of large-scale (country-wide) deployments. Three examples are given: Portugal, Italy and Norway.

In Portugal, it was the incumbent itself deciding to start rolling out FTTH, in order to stay competitive vis-à-vis the cable operator in the digital television market. This case leads to interesting conclusions, as it is often said that the only application that truly needs FTTH networks, is video. Cable operators are ahead because their networks are built to transmit video-services, and they can more cost efficiently upgrade their network bandwidth as well. Clearly this illustrates that there is no such thing as a "killer app", but the use of multiple high-quality video-related services simultaneously is a good motivation to begin to deploy FTTH. Note that the case in Portugal is very similar as what is happening in many Eastern European countries where incumbents are starting with FTTH rollouts due to a lack of good infrastructure.

In Italy, a new company was set up: Fastweb. They saw opportunities in connecting residents in seven municipalities (cities like Milan and Rome and their environments), where the Internet conditions were rather rudimentary. Formulating a partnership with AEM, the electricity company in Milan, they were able to save costs on digging, and in turn gained additional subscribers for the network.

The third large-scale deployment was initiated and fully deployed by Altibox, a subsidiary of the regional Norwegian energy supplier Lyse Energi. This again is a completely different case, with a utility company opting for a "multi-utility" strategy, offering both energy and broadband.

Although the initiators for the three large-scale deployments under study are very diverse, the business model used is the same: a Vertically Integrated model in which one company is responsible for both the passive and the active infrastructure, as well as for the provisioning of the services.

\section{Rural Regions}

Rural areas are characterized by rudimentary access to the Internet, and broadband DSL or cable networks are not available everywhere. One could conclude that rural 
areas form good markets to start deploying FTTH, wouldn't it be that the upfront costs are much higher than in urban areas (because the distances to be bridged in between two homes are much higher). Because of this high upfront investment, and lack of interest from the incumbents to invest, the initiative to deploy the network was always taken by a public institution: a public utility firm or the municipality itself.

Furthermore, it is important to mention that rural areas with no other broadband infrastructure available are the only areas where public funding is allowed (the socalled white areas, as defined in the European Regulatory framework [6]). This again discourages private firms to invest in FTTH in rural regions.

\section{Benefits from a Holistic Approach}

It is remarkable to see that in almost all cases, it wasn't the incumbent operator that had deployed the network, but a third party. For most European operators, there is more value in upgrading their existing infrastructure than installing a new FTTH network, regardless of the higher operational expenditures. But as soon as one operator deploys FTTH, all other operators will most probably follow in order not to lose their foothold [1].

Therefore, it is important to look for methods to improve the FTTH business case. One approach to improving the FTTH business case could be to use a holistic approach, tackling the business case on three fronts - strategic geo-marketing, synergetic installation and detailed operational modeling, which is the topic of the next paragraph and is based on [2].

\subsection{Focus on the Best Customers}

The operator needs to know which areas in a region to roll-out first and which areas to postpone or skip. The customer is of vital importance in the outcome of the business case, and the operator will have to select the best set of customers to connect in order to maximize its business case. Working at this level requires a huge amount of information and calculation, and this quickly becomes prohibitive. Building such a marketing strategy requires intelligent clustering approaches aimed at reducing the complexity while not discarding too much detail.

Figure 2 shows the three steps in constructing the best cherry picking strategy for the operator, using both user related information (like demographic, economic and marketing information) and infrastructure related information (e.g., geographic information and information about existing infrastructure). The first step includes gathering all information and classifying all inhabitants according to a limited set of profiles. Secondly, the customers should be grouped in selected areas according to geographical distance and their assumed ARPU as found in their profile. As such we will most probably find different smaller closed areas in the region in which all customers have (or lack) more or less the same drive towards FTTH. Finally, at the end of the first two steps, the operator ends up with a data-set containing for each customer its geographical group and customer profile it belongs to. This information will 


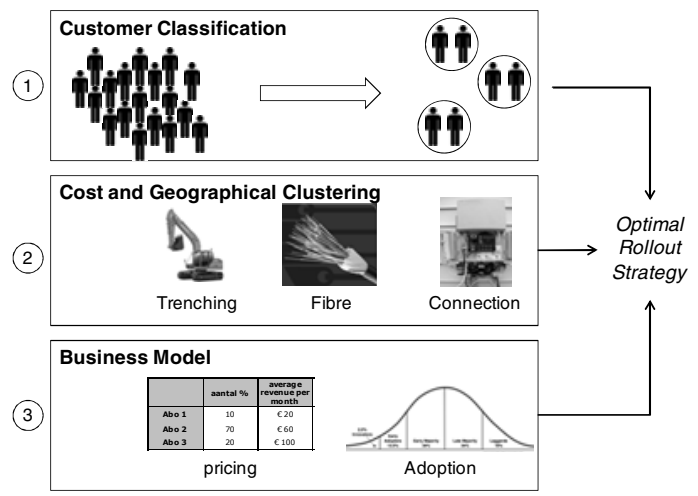

Fig. 2. The three geo-marketing steps for an optimal rollout

form the basis for calculating the cost for the deployment of FTTH in each part of the region. Deploying each group at the right time, taking into account the full business case, will provide the optimal roll-out strategy for the considered region.

\subsection{Deploy in Synergy}

The cost of installing fibers in the network, also referred to as the outside plant, will dominate (e.g., in a fully buried installation this can amount up to $70 \%$ of the overall costs). The business case can be substantially improved by lowering this installation cost. The largest reduction in cost can be achieved by finding synergies with other infrastructure owners for the installation of the network, as shown in Figure 3.

It is worth noting that the synergy can stretch up to the customer connection, in which the customer is connected to all infrastructures in only one intervention. Cooperation between the different infrastructure owners will reduce the final costs charged to the customers as well as reduce the amount of road works in the area.

\subsection{Operate the Joint Infrastructure}

Installing the outside plant in cooperation with other operators and infrastructure owners will definitely reduce the costs for each actor. On the other hand, such joint installation will undoubtedly lead to important questions considering the operations of the network. Operational expenditures can be up to $50 \%$ of the total costs, yet they are often modeled with little or no detail. This uncertain situation poses additional risks.

In order to accurately model the operational costs, two aspects are essential: the flow of activities in the considered processes needs to be detailed and the required input data has to be estimated. It is advisable to use a modeling language (or graphics) that is intuitive to the different people involved in the process (e.g., technicians, experts). Typically flowchart-based approaches are used for this case. 


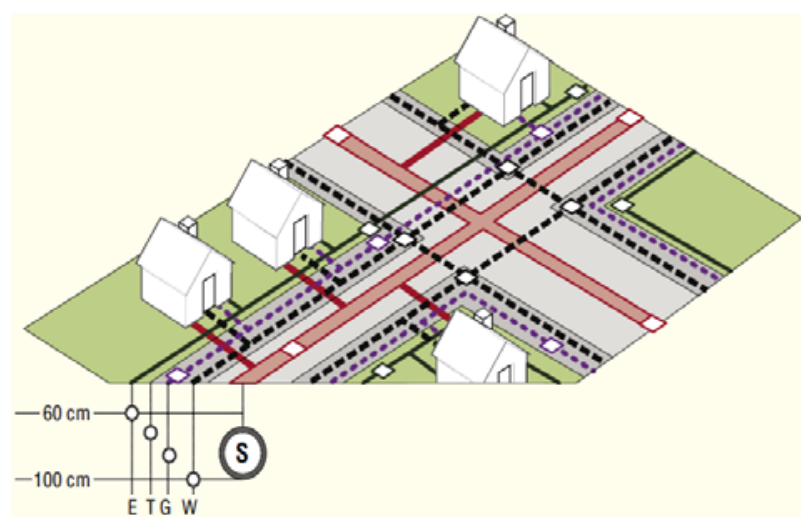

Fig. 3. The short proximity of underground infrastructures (Electricity (E), Telecom (T), Gas (G), Water (W) and Sewage (S))

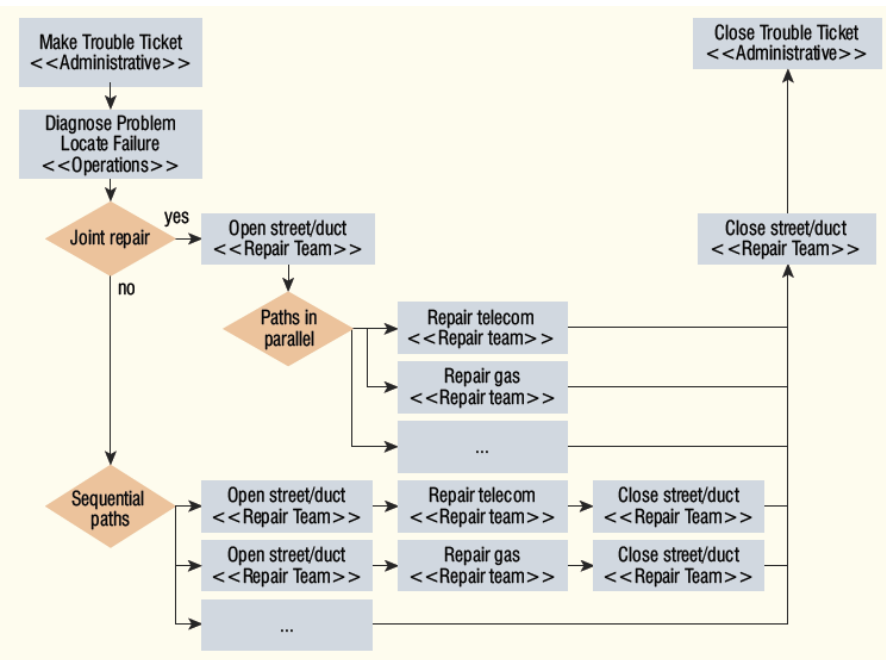

Fig. 4. Flowchart model of the repair process with an indication of the difference between joint and separate repair actions

Figure 4 gives an example for the process model used [1]. This process model shows the actions to be taken when an underground infrastructure is damaged. This model was used as a starting point and a quantitative cost comparison was made between a separate repair process and a joint repair process for all infrastructures telecommunications, electricity, gas and water. The results showed that, when infrastructures are not close to each other and consequently do not fail together often, this joint process would increase the operational expenditures for the repair. On the other hand, when the infrastructures are close to each other and have a larger possibility for failing together, the joint process can become more cost-effective. 
In the situation where the infrastructures always fail together, a cost saving of the repair process (up to $40 \%$ ) is possible.

It is essential for an operator to model all operational processes in detail. Once modeled, the costs for executing these processes can be estimated quite straightforward. More detailed modeling of all operational processes will also enable the operator to compare trustworthy, different alternative installation types or operations, to find bottlenecks and scheduling problems. As such he can keep a good eye on the current OpEx and control and optimize future OpEx.

\section{Game-Theoretic Approach to Improve Results and Conclusions}

A third and final issue that will be tackled in this section is the impact of competition with the existing telecom operators on the viability of an FTTH network rollout. Operators do not act in a monopoly environment and must consider the consequences of their actions and decisions on the behavior of their competitors. To model the competitive behavior, game theory is very well suited. Game theory is "aimed at modeling situations in which decision makers have to make specific actions that have mutual, possibly conflicting, consequences" [3]. When it is assumed that all players play at the same time and have sufficiently good knowledge of each other's strategies and payoffs, the game can be represented by means of a payoff matrix. In this matrix, each player has a payoff for all strategic combinations represented in the game. This allows to use tools to find equilibrium states in the game. In an equilibrium state, no player is inclined to change its strategy.

The most commonly known equilibrium state is the Nash Equilibrium (NE), which is defined as the strategy combination in which no player is inclined to unilaterally change its strategy. It is assumed that a game with fully rational players is expected to result in one of the NEs of the game being chosen. Typically static games (the game has one stage in which the players interact) can also be reduced or solved by removing strictly dominated strategies. These dominated strategies have a strictly lower payoff than another (dominant) strategy for all possible counterstrategies. No fully rational player would play a strictly dominated strategy but would instead play the dominant strategy. As such this strategy can be removed for the considered player. By iteratively using this approach for the different players, the matrix of the game can be simplified and can in some case be solved to a unique strategic choice for each player. In these cases, this strictly dominant strategy set is the only NE of the game.

To use the concepts on game theory, it is important to model the impact of the competition between all players on their respective business cases. Most likely, the chosen strategies will have an impact on the division of the market between all players, which in turn will have a significant influence on the potential revenues for each competing operator. In [1], where the strategies consisted of rollout speeds of the different fixed networks, a first mover advantage was introduced to divide the potential market between the cable operator and the FTTH network. Being the first to offer services to consumers resulted in a faster adoption and larger initial market share. 
In another case, using game theory to model the competition between different wireless operators, price competition was introduced to divide the market between the different operators [7].

Using game theory and an adequate competition model allows the analysis of the business case of an FTTH deployment under more realistic assumptions, especially since the viability of such a deployment largely depends on the take rate and as such the competition with other operators. If the FTTH network is rolled out in regions where existing communication infrastructures, like DSL and/or cable, are present, this could drastically impact the economic assessment. Applied to the specific case of a municipality rolling out an FTTH network where an existing cable operator upgrades its network to DOCSIS 3.0, game theory offers insight in the most profitable rollout scenarios under competition. Since an FTTH rollout suffers from high upfront installation costs, it is advantageous to start with a slower rollout compared to the cable operator and focus first on the largest industrial sites. In residential areas, FTTH should focus on the densest regions, since this reduces the digging costs per connection. On the other hand, the cable operator has less digging constraints and focuses on the larger residential areas.

\section{Conclusions}

Broadband penetration is continuously increasing in European countries and operators have to face end users' demand for higher bandwidth. Fiber optical networks are considered as the most future-proof access technologies, but the upgrade towards FTTH is happening much slower than expected. Apart from huge investments needed for fiber deployment other issues improve the viability of the FTTH business case.

First, a framework was proposed to identify different business models: the network matrix. It contains three axes: network lifecycle phases, network segments and network layers, from which the three main roles in an FTTH network deployment can be subtracted: the Physical Infrastructure Provider, the Network Provider and the Service Provider. Several European cases were analyzed and compared, to come to the main conclusion that it most of the existing network deployments, it is a third party taking the initiative for fiber deployment (like the city or a utility network owner). The business models applied vary from being vertically integrated to completely open (for competition).

Although incumbents are currently not investing in FTTH deployments, it is beyond doubt that, if one operator starts deploying the network, others will soon follow in order not to lose too many customers. Therefore, it is important to try to minimize the costs for deployment and maximize the efficiency. This can be done by using a holistic approach, focusing on three steps. First, the operator should subdivide the customers and first connect the most profitable ones. Secondly, synergies with other utility network owners can be agreed upon to reduce the cost for the outside plant deployment. And finally, it is important to not only look at the CapEx, but also model the OpEx in enough detail, so that these costs will not come as a surprise when the network is deployed. 
The last subsection of this chapter explained how to investigate the impact of competition on the market share (and thus revenues) that each of the operators can obtain (analysis based on GT). This is especially important to investigate when an FTTH network is deployed in an area where other (copper or cable) networks are already present. Results from a case study showed that when installing a FTTH network in such a region, the deployment should first focus on the densest regions, since this will entail the highest revenue potential.

Open Access. This article is distributed under the terms of the Creative Commons Attribution Noncommercial License which permits any noncommercial use, distribution, and reproduction in any medium, provided the original author(s) and source are credited.

\section{References}

1. Casier, K., et al.: Game-Theoretic Optimization of a Fiber-to-the-Home Municipality Network Rollout. Journal of Optical Communications and Networking 1(1), 30-42 (2009)

2. Casier, K., et al.: Improving the FTTH Business Case. Benefits of a holistic approach. The Journal of the Institute of Telecommunications Professionals 5(1), 46-53 (2011)

3. Felegyhazi, M., Hubaux, J.P.: Game theory in wireless networks: a tutorial. EPFL Technical report, LCA-REPORT 2006-002 (2006)

4. Forzati, M., Larsen, C.P., Mattsson, C.: Open access networks, the Swedish experience. In: International Conference on Transparent Optical Networks (ICTON), Munich, Germany (2010)

5. Kurose, J.F., Ross, K.W.: Computer Networking: A top-down approach featuring the Internet, 4th edn. Addison Wesley (2008)

6. Queck, R., et al.: The EU Regulatory Framework Applicable to Electronic Communications. Telecommunications, Broadcasting and the Internet: EU Competition Law and Regulation (3rd Revised edition). S.a. Maxwell, London (2010)

7. Tahon, M., et al.: Municipal support of wireless access network rollout: a Game Theoretic Approach. Telecommunications Policy 35(9-10), 883-894 (2011)

8. Van der Wee, M., et al.: Measuring the success rate of fiber-based access networks. Evaluation of the Stokab case and comparison to other Western European cases. Institute of Telecommunications Professionals 5(4), 22-31 (2011)

9. Verbrugge, S., et al.: Research Approach towards the Profitability of Future FTTH Business Model. Future Network and Mobile Summit, Warsaw, Poland (2011) 